\title{
L2 Lexical Attrition and Vocabulary Re-learning in Three L1 English L2 Japanese Children
}

\author{
Jenifer Larson-Hall \\ Kitakyushu University \\ doi: http://dx.doi.org/10.7820/vli.v06.2.Larson-Hall
}

\begin{abstract}
In this paper I review the evidence for the role of age in affecting second language attrition, and find it strongly supports a large difference in attrition around a breakpoint of age 8. I propose a Dynamic Attrition Model which posits that attrition sets in immediately upon the loss of contact with a language but the speed of loss differs depending on age. Three children who began their incubation periods at age 6,8 and 10 are examined when they are re-exposed to Japanese 6 years later. All children showed strong savings rates but large losses to their L2 Japanese.
\end{abstract}

This paper will examine the topic of L2 lexical attrition by situating a case study of three language attriters within a theoretical framework about the process of L2 lexical loss which I shall call the Dynamic Attrition Hypothesis.

After about 30 years of research on how second language learners lose (attrite) their L2 vocabulary, there are a few basic findings that can be stated with certainty. The first is that age plays a very strong role in the strength of language attrition. Learners whose age is under about 8 or 9 (Yoshitomi, 1992) when contact with an L2 is cut off completely have been reported to have catastrophic language attrition, while adult learners generally do not show much evidence of loss in the sense of tests on discrete vocabulary. Here I will define catastrophic language attrition as a situation where the language learner is not able to communicate productively (beyond a few formulaic sequences) in the language, and on tests of discrete vocabulary words scores no higher than $50 \%$ (of initially known words) on receptive tests or $25 \%$ on productive tests.

Catastrophic language attrition has been found with learners aged 8 or younger in Burling (1978), Hansen (1980, cited in Hansen \& Reetz-Kurashige, 1999), Hansen-Strain (1990), Ioup (2001), Kuhberg (1992), and Yukawa (1997). All of these studies reported that children who had been fluent and proficient speakers in an L2 became unable to communicate in the language. Studies of very young children aged 5 or younger who were followed systematically from the onset of attrition (and in all these cases the children were the author's children) were found to lose all productive ability by at most 6 months (Burling, 1978; Hansen-Strain, 1990; Yukawa, 1997). There are other studies with children aged 8 or younger which did not find catastrophic loss but all of these found evidence of severe loss of productive vocabulary ability; most such studies were done with children at the upper threshold of the age limit and over incubation periods (times when the language contact was discontinued) of at most 1 year (Cohen, 1986; Olshtain, 1986, 1989). 
For adults and older children, however, the situation is quite different and many studies have found almost no attrition at all (Mehotcheva, 2010; Murtagh \& van der Slik, 2004; Olshtain, 1986; Russell, 1999; Taura, 2008; Tomiyama, 1999; Weltens, Van Els, \& Schils, 1989; Xu, 2010; Yoshitomi, 1999). However, most of the studies of adult attrition have involved incubation periods of around 1-2 years, with only a few going as much as 4 or 5 years, and in these cases some language contact was maintained. Most of the studies which have been done with cases of longer incubation periods involve Mormon missionaries and are cross-sectional, assuming that the former missionaries would have known certain words given the nature of their language immersion experience. However, Graham (2012) longitudinally studied $N=12$ former missionaries who spent 24 months abroad after 12 years of incubation and reported significant losses in the number of tokens used on a narrative task, but all of the participants were still able to function in their L2 Spanish.

On the contrary, for much longer incubation periods, some authors have documented catastrophic language attrition for adults. Ioup (2001) tested a child who spoke Arabic from age $0-13$ but then had 45 years without any contact. Ioup reports that this former L2 Arabic speaker had no proficiency in the language at the time of testing, but did show some signs of retention as evidenced by the fact that he had a medium savings ratio in relearning Arabic words (meaning that he remembered real Arabic words better than nonsense words he was taught during an experimental phase). This so-called "savings" effect, first noted by Nelson (1978), indicates some kind of residual knowledge of a word in spite of not being able to recall it productively or receptively. Hansen (1999) tested missionaries with incubation periods of 30 years and reports that half of the L1 English L2 Japanese female missionaries she tested, who had lived in Japan for 24 months, could not do a story-retelling task at all and could only spontaneously produce numbers and a few formulaic expressions, while all of the male missionaries, who had lived in Japan for a longer period of 3 years, were able to do so. Hansen et al. (2012) tested L1 English speaking former missionaries of L2 Spanish, Portuguese, German, Mandarin, Japanese, and Korean by phone and asked them to produce the target word after hearing each word spoken in English twice. Participants were tested over a range of incubation periods from 0 to 50 years, and results show that after about 10 years there is a modest amount of catastrophic loss (productive vocabulary score $<25 \%$, but only among speakers of L2 Japanese, Korean, or Mandarin.

It is, thus, clear that while age plays an outsized role in the extent of L2 lexical attrition, other factors such as the highest language proficiency attained when a person starts the attrition period, the length of the incubation period, and language distance do play some role as well in explaining how quickly catastrophic language attrition occurs. However, I believe the same basic mechanism of loss underlies all L2 lexical attrition although the speed of loss seems to depend on the age of attrition. Whether child or adult, this hypothesis provides an explanation for why attrition can be discovered in some cases but not in others. Basically, I hypothesize that lexical attrition sets in immediately following the loss of language contact and results in continuous language attrition events, but it does not become clearly evident until a catastrophic attrition loss occurs.

The idea of continous attrition events that may not become evident until a sudden collapse of a system was detailed by Meara in two papers that described a 
thought experiment (1989) and then a computer simulation (2004) of a very simplified lexical system. Meara (2004) sets up a 2500-word vocabulary system where each word is linked with two other words. Each word has a property of being activated either when both words it is linked to are activated (AND words) or being activated when just one of the words it is linked to is activated (OR words). Randomly implementing the properties of each word, the system rapidly settles into a stable state where most words are either permanently activated (available productively) or deactivated (available only receptively), with only a small number flickering between the two states. Meara (2004, p. 141) states, "this is a very important property, because it suggests that lexical attrition is NOT a property of individual words that make up a lexicon; rather, it is a property of the network of relationships between the words." In order to model lexical attrition in this simplified system, Meara programs words to be randomly demoted from those that are activated if only one of the two links is active (OR words) to those that are activated only if both of the links are active (AND words).

In running this simulation multiple times, Meara obtains a set of cases of what could possibly occur in such a system. In most cases, the system experiences attrition events and though there may be some small loss of activated vocabulary, the system seems fairly stable until it suddenly and catastrophically collapses and the number of activated units drops to close to 0 .

Robert Nelson (personal communication) notes, however, that Meara's model is wrong; it sets up a system where every word in the network is connected to the same number of words, which is unrealistic given that some words, such as function words or determiners, will have many more connections than will content words. Given that a better model of a human vocabulary system is one where the number of connections is relatively few for most words but can be quite high for a small number of words (meaning the distribution of a lexical network follows Zipf's law) more than $50 \%$ of the connections would have to be deleted before catastrophic loss can be detected. Nelson (personal communication) proposes that each word has its own memory strength that is proportional to the number of times that the word has been used.

One interesting aspect of both Meara's and Nelson's simulations is that even though the rate of attrition events (loss) is assumed to be continuous across all of the iterations, the loss of the system (or the word, for Nelson) happens quite quickly, not gradually (see Figure 1). In Meara's system, the loss occurs at a random point,
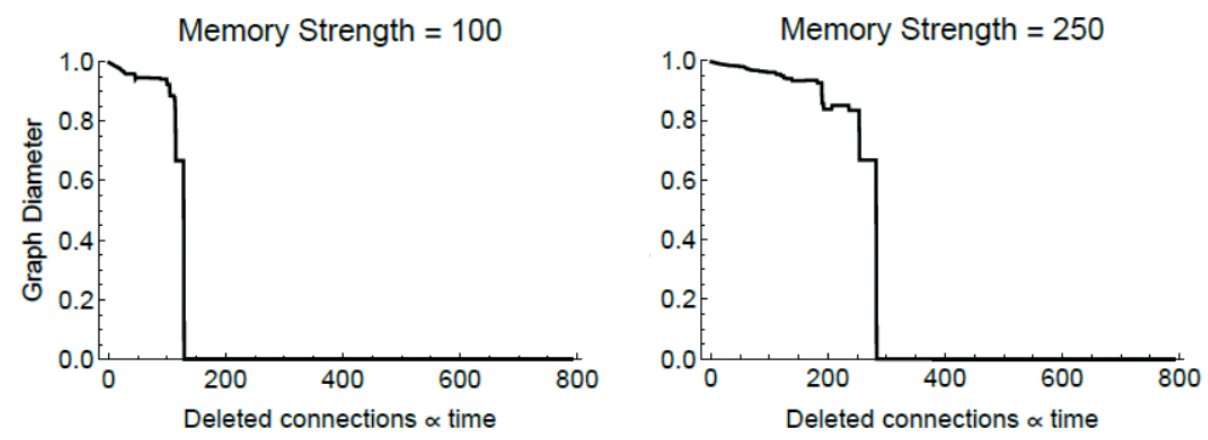

Figure 1. Nelson's proposal regarding loss of individual words, depending on the number of times the word has been used (memory strength) (personal communication). 
sometimes after 100 and sometimes after 250 iterations. On the contrary, for Nelson's idea the more times a word has been used the more time it takes to be lost. However, in both cases the loss is quite sudden. We may have an image in our minds of lexical loss as the leaking away of one word at a time, like water dripping from a hole in a paper cup. If these simulations can indeed model the way human loss works, they would indicate that a better metaphor of loss would be of a tree branch that has been stressed over time finally breaking off the tree. The fracture may be invisible or mostly so until the damage becomes so great that the result is catastrophic.

In applying the findings of the simulated vocabulary network to real-world attrition, Meara hypothesizes that attrition events in the model (demotion of AND words to OR words) are similar in real life to deactivated words that are available receptively but not productively (2004, p. 139). Meara hypothesizes that these words can be re-activated so that attrition events do not necessarily equal the loss of a word forever. Even though Nelson's model is undoubtedly much more likely to simulate real human vocabulary networks, Meara's ideas about activation may still hold. Meara says this simulation is modeled on a dynamic systems approach and has a lot in common with connectionist models. I have, therefore, labeled it the Dynamic Attrition Model.

Such a system, applied to real life attrition cases, would explain why studies of adults find that there is very little attrition over the short-term and why vocabulary knowledge seems stable even over decades of incubation (Bahrick, 1984a, 1984b), but very long-term studies such as those cited above by Hansen show evidence of catastrophic language loss. However, with children the situation seems to be different and the decay occurs on a faster scale. In order to know more about how age affects L2 lexical attrition, we need to examine more cases of language learners who are close in age to the cut-off point of age 8 or 9 when their incubation period starts. Although the Dynamic Attrition Model theory does not actually make a difference in the kind of testing that we do (there is not much alternative to testing for individual vocabulary words or overall speaking ability), results will be interpreted in this framework.

This study reports on the L2 lexical language attrition of three L1 English siblings who lived with their L1 English-speaking American parents (the D family) for 3 years in Japan from the ages of 3;7-6;7 (Elizabeth), 5;4-8;4 (Matthew), and 7;7-10;7 (Holly) (all names are pseudonyms). Their family then moved to the United States for 1 year, then Nepal and Kenya (their father was in the foreign service). The children returned to Japan approximately 6 years later at ages 12;8, 14;5, and 16;8, whereupon their residual Japanese ability was tested. Their ages at the start of the 6-year incubation period were, thus, below the hypothesized cutoff point (age 6), right around the cut-off point (age 8), and right above the cut-off point (age 10). Besides the three siblings, one pair of additional siblings from the M family (L1 English, both parents American) who attended the same school and church and, thus, would be expected to have similar levels of input in English and Japanese, but who had previously never been exposed to Japanese, were also tested. JC was 12;7 at the time of testing and Kitty was 9;7.

A 3-hour battery of tests was administered to all participants but in this report I will focus on three tests which either tested specific vocabulary words or showed a comprehensive speaking ability. There were two tests of comprehensive 
speaking ability; one was a picture book narration and the other was an interview with a native-speaking Japanese adult. The one test of specific vocabulary words was a savings test, looking for residual knowledge of inaccessible words. The participants were asked to name concrete nouns from picture cards and were tested until each had reached 16 real words - which they did not know. These real words were randomly mixed with 16 nonsense words, and all words were taught orally to each participant. After 20-30 minutes, the participant was tested on all the words and a ratio of real words remembered to nonsense words was calculated.

The results showed that for both the interview and picture book narration, the two younger attriters (Elizabeth and Matthew) showed catastrophic attrition loss, very similar to JC and Kitty who could not perform at any level beyond extremely simple formulaic sequences on either activity. On the contrary, Holly was able to understand simple questions in the interview although she had forgotten most of the concrete vocabulary outside of formulaic sequences. For example, she could understand and answer a question about her name and age, but when asked if she liked to cook, she knew it was a question about whether she liked something but couldn't understand the word for cooking, nor could she answer the question in Japanese once she understood the word. In the picture book narration, Holly was able to say very simple phrases although she often lacked specific vocabulary words she needed, or thought she knew the right word but actually used an incorrect word. She had certainly lost a large amount of proficiency in Japanese in her incubation period but did retain the ability to put together sentences with the vocabulary she remembered.

As concerns the savings test, the number of words tested until 16 unknown words were reached was 50 for Holly, 19 for Matthew, 22 for Elizabeth, 21 for JC, and 17 for Kitty. Their savings ratios (nonsense words/real words) were 1/4 for Holly, 1/5 for Matthew, 0/4 for Elizabeth, and 0/0 for both JC and Kitty. According to comparative data from Hansen, Umeda, and McKinney's (2002) synthesis of savings tests, all of the D family children had strong savings strength while the savings strength for the $\mathrm{M}$ family was non-existent.

In summary, the results of the testing with the D family showed that the children who had been aged 8 or younger when their 6-year incubation period began had experienced catastrophic loss of their Japanese language ability in that time period, although savings tests indicated evidence that some memory traces of their ability remained. The oldest child of the $\mathrm{D}$ family, however, was able to retain a low level of functionality in using Japanese although her vocabulary had significantly atrophied. It thus appears that in this case, the cut-off age of 8 or 9 is an accurate point at which the pace of the dynamic lexical L2 attrition may change dramatically.

\section{References}

Bahrick, H. (1984a). Fifty years of second language attrition: Implications for programmatic research. The Modern Language Journal, 68, 105-118. doi:10.1111/j.1540-4781.1984.tb01551.x

Bahrick, H. (1984b). Semantic memory content in permastore: 50 years of memory for Spanish learned in school. Journal of Experimental Psychology: General, 113, 1-29. doi:10.1037/0096-3445.113.1.1 
Burling, R. (1978). Language development of a Garo- and English-speaking child. In E. Hatch (Ed.), Second language acquisition (pp. 54-75). Rowley, MA: Newbury House.

Cohen, A. (1986). Forgetting foreign-language vocabulary. In B. Weltens, K. de Bot, \& T. van Els (Eds.), Language attrition in progress (pp. 143-158). Dordrecht: Foris.

Graham, C. R. (2012). Vocabulary attrition in adult speakers of Spanish as an L2. In L. Hansen (Ed.), Second language acquisition abroad: The LDS missionary experience (pp. 135-184). Amsterdam: John Benjamins.

Hansen, L. (1999). Not a total loss: The attrition of Japanese negation over three decades. In L. Hansen (Ed.), Second language attrition in Japanese contexts (pp. 142-153). New York: Oxford University Press.

Hansen, L., Colver, A., Chong, W., Pereira, H., Robinson, J., Sawada, A., \& Miller, R. M. (2012). The lost word: Vocabulary attrition in six mission languages. In L. Hansen (Ed.), Second language acquisition abroad: The LDS missionary experience (pp. 111-134). Amsterdam: John Benjamins.

Hansen, L., \& Reetz-Kurashige, A. (1999). Investigating second language attrition: An introduction. In L. Hansen (Ed.), Second language attrition in Japanese contexts (pp. 3-18). New York: Oxford University Press.

Hansen, L., Umeda, Y., \& McKinney, M. (2002). Savings in the relearning of second language vocabulary: The effects of time and proficiency. Language Learning, 52(4), 653-678. doi:10.1111/1467-9922.00200

Hansen-Strain, L. (1990). The attrition of Japanese by English-speaking children: An interim report. Language Sciences, 12(4), 367-377. doi:10.1016/0388-0001(90)90004-Z

Ioup, G. (2001). Exploring age and loss using the savings paradigm. Paper presented at the AAAL, Saint Louis, MO.

Kuhberg, H. (1992). Longitudinal second language attrition versus second language acquisition in three Turkish children: Empirical findings. Second Language Research, 8, 138-154.

Meara, P. (1989). Matrix models of vocabulary acquisition. AILA Review, 6, 66-74. Retrieved from http://www.aila.info/download/publications/review/ AILA06.pdf

Meara, P. (2004). Modelling vocabulary loss. Applied Linguistics, 25(2), 137-155. doi:10.1093/applin/25.2.137

Mehotcheva, T. (2010). After the fiesta is over: Foreign language attrition of Spanish in Dutch and German Erasmus students. Unpublished doctoral dissertation, University of Groningen.

Murtagh, L., \& van der Slik, F. (2004). Retention of Irish skills: A longitudinal study of a school-acquired L2. International Journal of Bilingualism, 8(3), 279-302. doi:10.1177/13670069040080030701

Nelson, T. (1978). Detecting small amounts of information in memory: Savings for nonrecognized items. Journal of Experimental Psychology: Human Learning and Memory, 4, 453-468. 
Olshtain, E. (1986). The attrition of English as a second language with speakers of Hebrew. In B. Weltens, K. de Bot, \& T. van Els (Eds.), Language attrition in progress (pp. 187-204). Dordrecht: Foris.

Olshtain, E. (1989). Is second language attrition the reversal of second language acquisition? Studies in Second Language Acquisition, 11, 151-165.

Russell, R. A. (1999). Lexical maintenance and attrition in Japanese as a second language. In L. Hansen (Ed.), Second language attrition in Japanese contexts (pp. 114-141). Oxford: Oxford University Press.

Taura, H. (2008). Language attrition and retention in Japanese returnee students. Tokyo: Akashi Shoten.

Tomiyama, M. (1999). The first stage of second language attrition: A case study of a Japanese returnee. In L. Hansen (Ed.), Second language attrition in Japanese contexts (pp. 59-79). Oxford: Oxford University Press.

Weltens, B., Van Els, T., \& Schils, E. (1989). The long-term retention of French by Dutch students. Studies in Second Language Acquisition, 11(2), 205-216. doi:10.1017/S0272263100000619

$\mathrm{Xu}, \mathrm{X}$. (2010). English language attrition and retention in Chinese and Dutch university students. GRODIL series (87). Unpublished $\mathrm{PhD}$ thesis, University of Groningen.

Yoshitomi, A. (1992). Towards a model of language attrition: Neurobiological and psychological contributions. Issues in Applied Linguistics, 3, 293-318.

Yoshitomi, A. (1999). On the loss of English as a L2 by Japanese returnee children. In L. Hansen (Ed.), Second language attrition in Japanese contexts (pp. 80-113). Oxford: Oxford University Press.

Yukawa, E. (1997). L1 Japanese attrition and regaining: Three case studies of two early bilingual children. Stockholm: Centre for Research on Bilingualism, Stockholm University. 